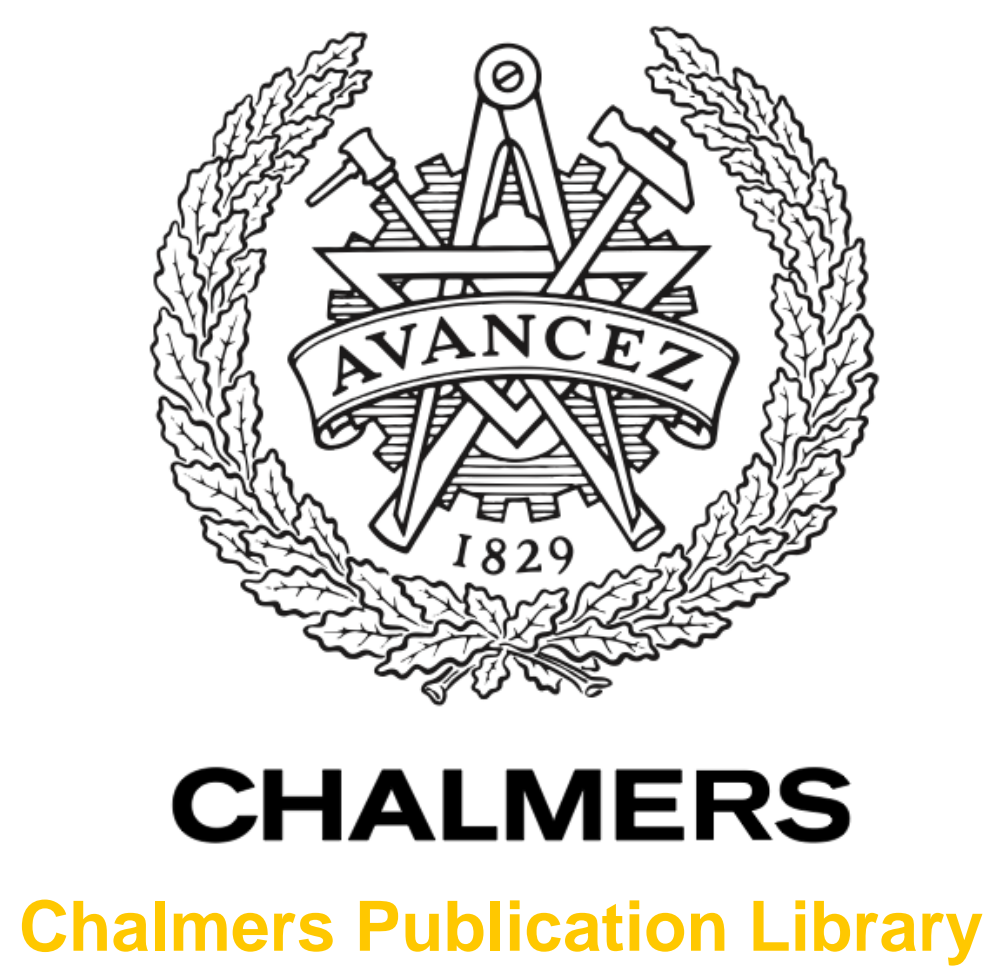

\title{
Linear and Nonlinear Crosstalk Tolerance of Polarization-Switched QPSK and Polarization-Multiplexed QPSK
}

This document has been downloaded from Chalmers Publication Library (CPL). It is the author's version of a work that was accepted for publication in:

\section{European Conference and Exposition on Optical Communications, ECOC 2011; Geneva;} Switzerland; 18 September 2011 through 22 September 2011 (ISSN: 21622701)

Citation for the published paper:

Sjödin, M. ; Johannisson, P. ; Andrekson, P. et al. (2011) "Linear and Nonlinear Crosstalk Tolerance of Polarization-Switched QPSK and Polarization-Multiplexed QPSK". European Conference and Exposition on Optical Communications, ECOC 2011; Geneva; Switzerland; 18 September 2011 through 22 September 2011

Downloaded from: http://publications.lib.chalmers.se/publication/141449

Notice: Changes introduced as a result of publishing processes such as copy-editing and formatting may not be reflected in this document. For a definitive version of this work, please refer to the published source. Please note that access to the published version might require a subscription. 


\title{
Linear and Nonlinear Crosstalk Tolerance of Polarization- Switched QPSK and Polarization-Multiplexed QPSK
}

\author{
Martin Sjödin, Pontus Johannisson, Peter A. Andrekson, and Magnus Karlsson \\ Photonics Laboratory, Department of Microtechnology and Nanoscience, Chalmers University of Technology, SE-412 96, Göteborg, Sweden \\ martin.sjodin@chalmers.se
}

\begin{abstract}
The OSNR requirements and linear crosstalk tolerance of PS-QPSK and PM-QPSK are investigated, as well as the transmission performance in the presence of $10 \mathrm{Gbit} / \mathrm{s}$ neighboring OOK channels.

OCIS codes: (060.1660) Coherent communications; (060.0060) Fiber optics and optical communications.
\end{abstract}

\section{Introduction}

Polarization-switched quadrature phase-shift keying (PS-QPSK) transmits three bits per symbol, where two bits are encoded in the QPSK symbol and the third bit in the switching between the polarization states. For two major reasons, this modulation format has received increasing attention recently. Firstly, while it is known that increasing the signal-space dimensionality (e.g. by using pulse-position modulation [1], or error-correcting codes) will lead to higher sensitivities, PS-QPSK has the highest sensitivity among 4-dimensional modulation formats. Compared to polarization-multiplexed QPSK (PM-QPSK), PS-QPSK has a sensitivity advantage of $0.97 \mathrm{~dB}$ at a BER of $10^{-3}$, and an asymptotic advantage of $1.76 \mathrm{~dB}$ [2]. Secondly, simulation results have been presented showing that PS-QPSK has higher nonlinear tolerance than PM-QPSK in WDM transmission scenarios [3,4]. These features make PSQPSK an interesting candidate for long-haul systems, such as transoceanic links. The first experimental results were presented in [5]. At $30 \mathrm{Gbit} / \mathrm{s}$, PS-QPSK required $0.7 \mathrm{~dB}$ lower OSNR than PM-QPSK to achieve a BER of $10^{-3}$.

In this paper, we present experimental results for 21 Gbaud PS-QPSK and make a comparison with PM-QPSK at the same bit rate $(63 \mathrm{Gbit} / \mathrm{s})$ and at the same symbol rate. We also measure the penalty from linear crosstalk in a WDM system at $25 \mathrm{GHz}$ and $50 \mathrm{GHz}$ channel spacing. Finally, in a transmission experiment over $320 \mathrm{~km}$ of standard single mode fiber (SSMF), we investigate how the two modulation formats perform in the presence of four neighboring on-off keying channels at $10 \mathrm{Gbit} / \mathrm{s}$ in a scenario where an OOK channel is upgraded.

\section{Experimental setup}

The PS-QPSK transmitter is shown in Fig. 1a. The light source was an external cavity laser (ECL) with $300 \mathrm{kHz}$ linewidth and the wavelength was set to $1554 \mathrm{~nm}$. PS-QPSK at $63 \mathrm{Gbit} / \mathrm{s}$ was generated in two steps: First, an IQ modulator (IQM) driven by two $21 \mathrm{Gbit} / \mathrm{s} 2^{15}-1$ PRBS generated Gray coded QPSK. In the second step, the QPSK signal was split into two branches, each containing a single-drive Mach-Zehnder modulator (MZM). The MZMs were driven in a push-pull configuration to switch the power between the polarizations. As a result, only one polarization per symbol slot had non-zero power after recombination with a polarization beam-combiner (PBC). To synchronize the on-off switching between the two data streams, the lengths of the fibers between the amplitude modulators and the PBC were adjusted to be the same. To have high correlation between the phase noise in the two polarizations, and thereby facilitating phase estimation after detection, it was also ensured that the total lengths of the two branches were approximately the same. PM-QPSK was generated with the transmitter shown in Fig. 1b. After the IQM the QPSK signal was split and recombined with a PBC with a relative delay $\Delta \mathrm{T}$ of about $10 \mathrm{~ns}$.

In a measurement of the tolerance to linear WDM crosstalk, two additional PS/PM-QPSK channels from other ECLs were used (shown by the dotted boxes in Figs. 1a and 1b) and the BER was measured for the center channel as a function of the OSNR and the WDM channel spacing.
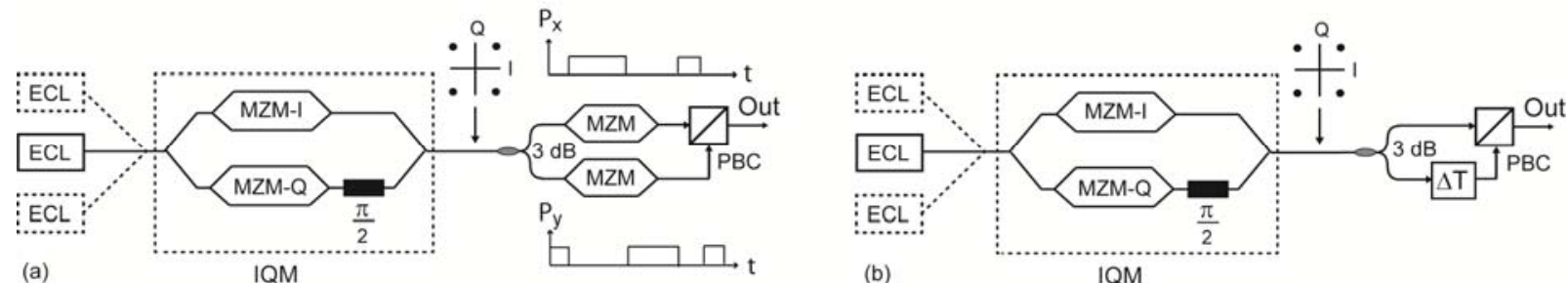

(b)

IQM

Fig. 1: (a) The PS-QPSK transmitter. The amplitude modulators are driven in a push-pull configuration to switch the power between the two polarization states. (b) The PM-QPSK transmitter. The delay $\Delta \mathrm{T}$ is about $10 \mathrm{~ns}$. 
The receiver is shown in Fig. 2a. The same hardware was used for both modulation formats. An attenuator (Att) and an EDFA with a noise figure of $5 \mathrm{~dB}$ were used to adjust the OSNR of the signal and a band-pass filter (BPF) with $1.2 \mathrm{~nm}$ bandwidth suppressed ASE noise outside the signal spectrum. Polarization diversity was obtained by splitting the signal with a polarization beam-splitter (PBS) and mixing the light in the x- and the y-polarization with the output from a local oscillator (LO) laser (an ECL with $500 \mathrm{kHz}$ linewidth) in two optical $90^{\circ}$ hybrids with integrated balanced detectors and electrical amplifiers. The combined $3 \mathrm{~dB}$ bandwidth of the detectors and the amplifiers was more than $25 \mathrm{GHz}$. After photodetection the signals were sampled synchronously at 50 Gsample/s by a real-time sampling oscilloscope with $16 \mathrm{GHz}$ analog bandwidth. The DSP algorithms for both PS-QPSK and PMQPSK are further described in [5,6]. The main difference between the DSP of the two formats is the polarization demultiplexing, since the constant modulus algorithm does not work for PS-QPSK.
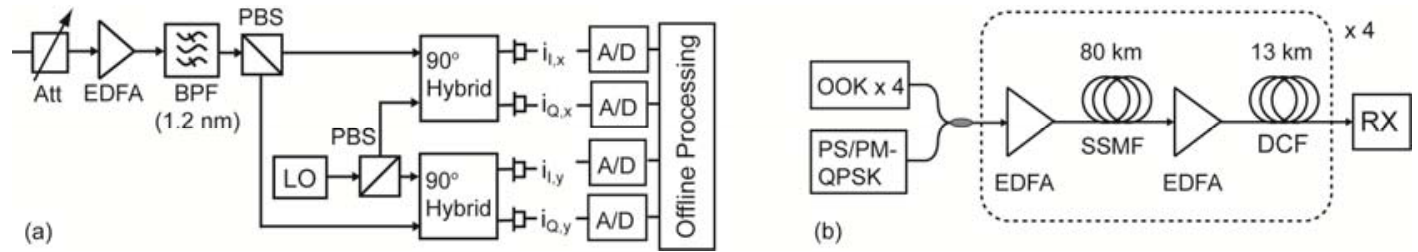

(b)

Fig. 2: (a) The receiver used to detect PS-QPSK and PM-QPSK. (b) The link used in the transmission experiment.

In the transmission experiment with OOK neighbors, the link shown in Fig. 2b was used. After each span of SSMF, the optical power was boosted with an EDFA and dispersion was compensated with dispersion compensating fiber (DCF), since this is commonly used in OOK systems. Similar to [5], the power into the DCF modules was set to be $5 \mathrm{~dB}$ lower than the SSMF launch power. Four OOK channels were generated by applying $10 \mathrm{Gbit} / \mathrm{s}$ PRBS data to an MZM modulating the output from four laser sources. The channel spacing was $100 \mathrm{GHz}$, with the PS/PMQPSK channel in the center. Since the aim was to investigate cross-phase modulation (XPM) effects, we set the power of each OOK channel to be three times larger than the power of the PS/PM-QPSK channel. For comparison, we also studied the single channel case for both PS-QPSK and PM-QPSK.

\section{Results}

The measured back-to-back BER as a function of OSNR for both PS-QPSK and PM-QPSK are shown in Fig. 3a. PS-QPSK requires $11.1 \mathrm{~dB}$ OSNR to obtain a BER of $10^{-3}$, compared to $12.1 \mathrm{~dB}$ for PM-QPSK at the same bit-rate. The difference of $1.0 \mathrm{~dB}$ in favor of PS-QPSK is in excellent agreement with the value predicted in [1] $(0.97 \mathrm{~dB})$. At the same symbol rate the measured difference between the two modulation formats is $2.1 \mathrm{~dB}$, which is very close to the theoretical prediction $(2.2 \mathrm{~dB})$. The analytical results from [2] has been included in Fig. 3a for the three cases. The measured PS-QPSK sensitivity is $1.3 \mathrm{~dB}$ worse than the theoretical prediction at the given BER of $10^{-3}$. For PM-QPSK, the differences are $1.3 \mathrm{~dB}$ and $1.2 \mathrm{~dB}$ at 15.75 Gbaud and 21 Gbaud, respectively. It can also be observed in Fig. 3 that the difference between the analytical and the measured BER curves is increasing slightly when the OSNR increases. The reason for this is intersymbol interference.
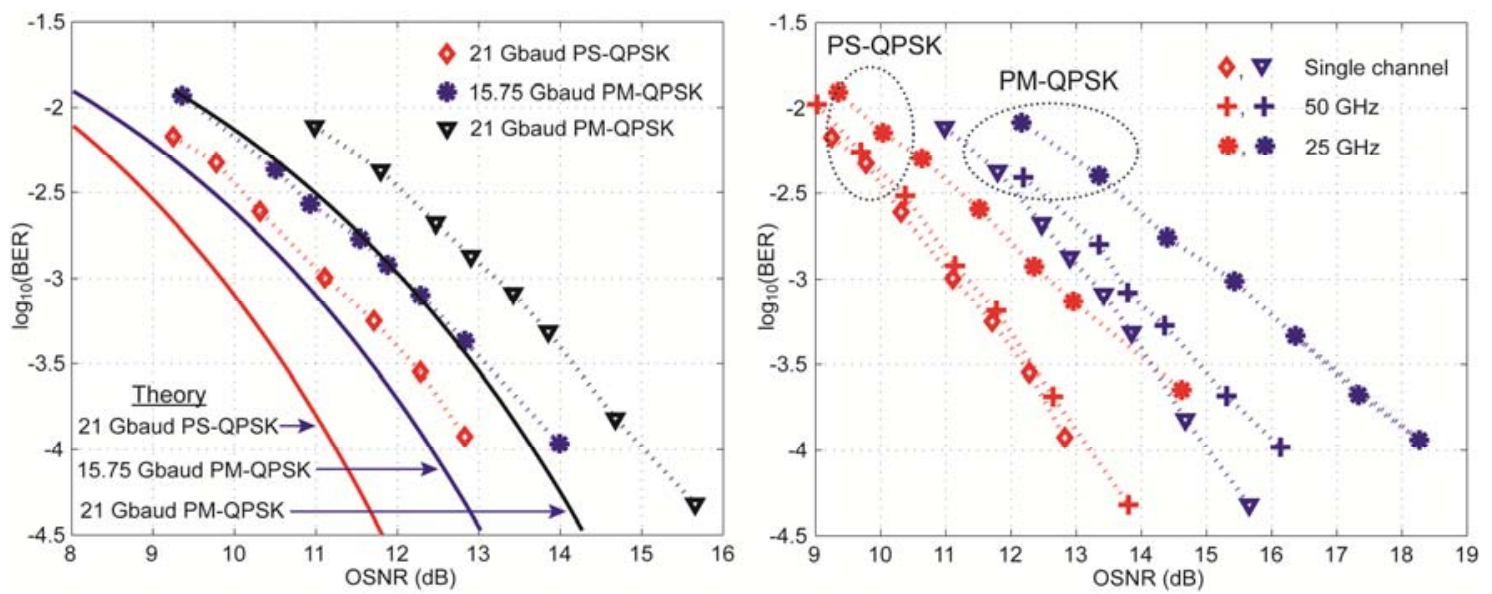

Fig. 3: (a) The measured back-to-back BER for 21 Gbaud PS-QPSK and PM-QPSK at both 15.75 and 21 Gbaud. The theoretical OSNR requirements are also shown for each case. (b) The measured back-to-back BER for 21 Gbaud PS-QPSK and PM-QPSK with two neighboring channels as a function of the OSNR and the channel spacing. 
Fig. $3 \mathrm{~b}$ shows the performance of the two modulation formats at 21 Gbaud when two neighboring channels are present at channel spacings of $25 \mathrm{GHz}$ and $50 \mathrm{GHz}$. The single channel performance is also included for comparison. For PS-QPSK, the OSNR penalties at a BER of $10^{-3}$ are $0.2 \mathrm{~dB}$ and $1.5 \mathrm{~dB}$ for $50 \mathrm{GHz}$ and $25 \mathrm{GHz}$ spacing, respectively. The corresponding penalties for PM-QPSK are $0.5 \mathrm{~dB}$ and $2.2 \mathrm{~dB}$, indicating that PS-QPSK has slightly higher tolerance to linear WDM crosstalk, even though both formats essentially have identical spectral envelopes. The spectral efficiency of 21 Gbaud PS-QPSK at $25 \mathrm{GHz}$ spacing is $2.35(\mathrm{bit} / \mathrm{s}) / \mathrm{Hz}$, with a 7\% FEC overhead taken into account.

Fig. 4a shows the transmission performance of the two modulation formats for the single channel case and in presence of the four neighboring $10 \mathrm{Gbit} / \mathrm{s}$ OOK channels. The OSNR required to obtain a BER of $10^{-3}$ is plotted against the total SSMF launch power, including the power of both PS/PM-QPSK and the OOK channels. The single channel performance for the three cases is similar. A $1 \mathrm{~dB}$ OSNR penalty is reached for launch powers of $8.2 \mathrm{dBm}$, $7.9 \mathrm{dBm}$ and 7.2 dBm for PS-QPSK, 15.75 Gbaud PM-QPSK and 21 Gbaud PM-QPSK, respectively. In presence of the OOK channels, the corresponding numbers are $5.9 \mathrm{dBm}, 4.2 \mathrm{dBm}$ and $4.8 \mathrm{dBm}$. This shows that at the same symbol rate, the tolerance to crosstalk from the OOK channels is similar for PS-QPSK and PM-QPSK, while the performance of PM-QPSK at 15.75 Gbaud is degraded more compared to the single channel case. Fig. $4 \mathrm{~b}$ shows the spectra (0.06 $\mathrm{nm}$ resolution) after the transmitter of the PS-QPSK channel and the four OOK channels.
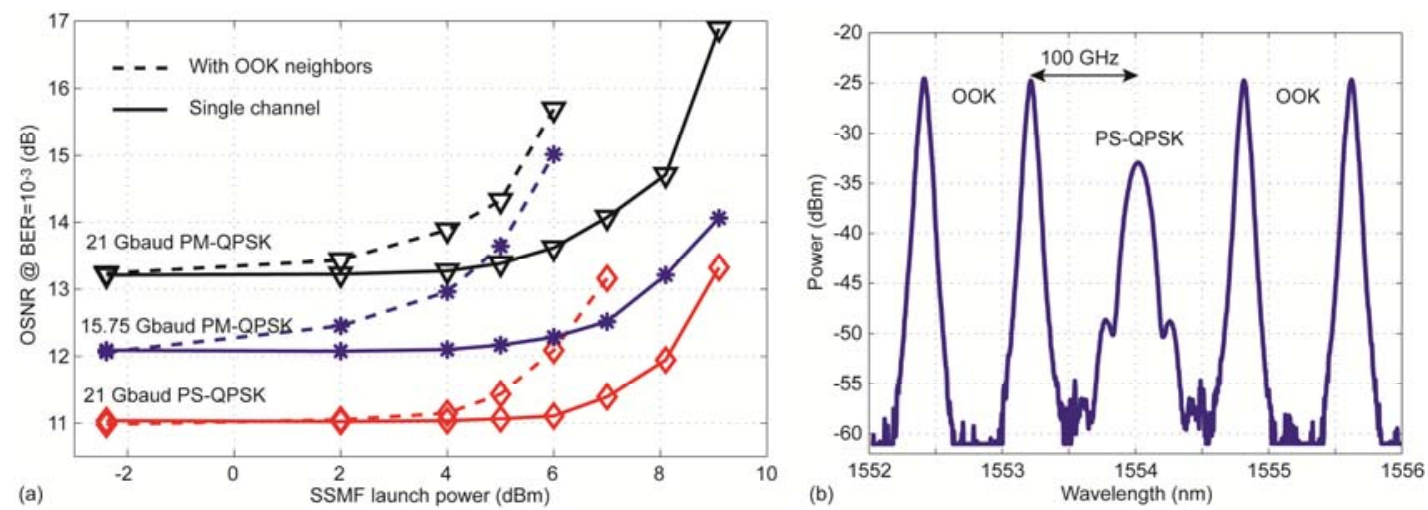

Fig. 4: (a) The required OSNR at BER $=10^{-3}$ for 21 Gbaud PS-QPSK and PM-QPSK at 15.75 and 21 Gbaud as a function of the launch power into the SSMF spans in the link. (b) Spectra after the transmitter showing the PS-QPSK and the OOK channels.

\section{Conclusions}

We have investigated the performance of PS-QPSK at $63 \mathrm{Gbit} / \mathrm{s}$ and compared with PM-QPSK at the same bit rate and the same symbol rate. The back-to-back measurements showed excellent agreement with theoretical predictions and PS-QPSK in a WDM system with a spectral efficiency of $2.35(\mathrm{bit} / \mathrm{s}) / \mathrm{Hz}$ resulted in only about $1.5 \mathrm{~dB}$ linear crosstalk penalty at a BER of $10^{-3}$. A transmission experiment over $320 \mathrm{~km}$ showed that at the same symbol rate, the two modulation formats have similar tolerance to XPM from four neighboring OOK channels, while the performance of PM-QPSK at the same bit rate as PS-QPSK was degraded more.

\section{Acknowledgement}

We acknowledge the financial support from the Swedish research council, the Vinnova IKT program, and the EU EURO-FOS project. This work was performed within the Fiber Optic Communications Research Center (FORCE) at Chalmers.

\section{References}

[1] X. Liu et al., "Demonstration of Record Sensitivity in an Optically Pre-amplified Receiver by Combining PDM-QPSK and 16-P. p.m. with Pilot-Assisted Digital Coherent Detection," in Optical Fiber Communication Conference. (OFC), p. PDPB1, 2011.

[2] M. Karlsson et al., "Which is the most power-efficient modulation format in optical links?," Optics Express, vol. 17, no. 13, pp. 10814$10819,2009$.

[3] P. Serena et al., "The performance of polarization switched-QPSK (PS-QPSK) in dispersion managed WDM transmissions," in European Conference on Optical Communication (ECOC), p. Th.10.E.2, 2010.

[4] P. Poggiolini et al., "Performance evaluation of coherent WDM PS-QPSK (HEXA) accounting for non-linear fiber propagation effects," Optics Express, vol. 18, no. 11, pp. 11360-11371, 2010.

[5] M. Sjödin et al., "Comparison of polarization-switched QPSK and polarization-multiplexed QPSK at 30 Gbit/s," Optics Express, vol. 19, no. 8, pp. 7839-7846, 2011.

[6] P. Johannisson et al., "Modified constant modulus algorithm for polarization-switched QPSK," Optics Express, vol. 19, no. 8, pp. 7734$7741,2011$. 